\title{
Decellularized allogeneic intervertebral disc: natural biomaterials for regenerating disc degeneration
}

\author{
Xianfeng Lin ${ }^{1,2, *}$, Xiangqian Fang ${ }^{1,2, *}$, Qiang Wang ${ }^{1,2, *}$, Zhijun $\mathrm{Hu}^{1,2}$, Kai Chen ${ }^{3}$, Zhi \\ Shan ${ }^{1}$, Shuai Chen ${ }^{1,2}$, Jiying Wang ${ }^{1,2}$, Jian $\mathrm{Mo}^{1,2}$, Jianjun Ma ${ }^{1,2}$, Wenbing $\mathrm{Xu}^{1,2}$, An \\ Qin ${ }^{4}$ and Shunwu Fan ${ }^{1,2}$ \\ ${ }^{1}$ Department of Orthopaedic Surgery, Sir Run Run Shaw Hospital, Medical College of Zhejiang University, Hangzhou, China \\ 2 Institute of Micro-Invasive Surgery of Zhejiang University, Hangzhou, China \\ ${ }^{3}$ Department of Orthopaedic Surgery, the First Affiliated Hospital of Wenzhou Medical College, Wenzhou, China \\ ${ }^{4}$ Department of Orthopedics, Shanghai Key Laboratory of Orthopedic Implant, Shanghai Ninth People's Hospital, Shanghai \\ Jiaotong University School of Medicine, Shanghai, China \\ * These authors have contributed equally \\ Correspondence to: Shunwu Fan, email: shunwu_fan@126.com
}

An Qin, email: dr_qinan@163.com

Keywords: intervertebral disc, decellularization, extracellular matrix, disc degeneration, mesenchymal stem cell, Pathology Section Received: November 26, 2015 Accepted: February 18, $2016 \quad$ Published: February 25, 2016

\section{ABSTRACT}

Intervertebral disc degeneration is associated with back pain and disc herniation. This study established a modified protocol for intervertebral disc (IVD) decellularization and prepared its extracellular matrix (ECM). By culturing mesenchymal stem cells (MSCs)(3, 7, 14 and 21 days) and human degenerative IVD cells ( 7 days) in the ECM, implanting it subcutaneously in rabbit and injecting ECM microparticles into degenerative disc, the biological safety and efficacy of decellularized IVD was evaluated both in vitro and in vivo. Here, we demonstrated that cellular components can be removed completely after decellularization and maximally retain the structure and biomechanics of native IVD. We revealed that allogeneic ECM did not evoke any apparent inflammatory reaction in vivo and no cytotoxicity was found in vitro. Moreover, IVD ECM can induce differentiation of MSCs into IVD-like cells in vitro. Furthermore, allogeneic ECM microparticles are effective on the treatment of rabbit disc degeneration in vivo. In conclusion, our study developed an optimized method for IVD decellularization and we proved decellularized IVD is safe and effective for the treatment of degenerated disc diseases.

\section{INTRODUCTION}

Intervertebral disc degeneration (IDD) is associated with back pain and disc herniation [1]. It is estimated that $84 \%$ of the people have experienced low back pain (LBP) during their lifetime, and the costs related to low back disorders exceed $\$ 100$ billion per year in the USA [2, 3]. IDD can usually lead to multiple spinal disorders such as disc herniation, spondylolisthesis and spinal stenosis [4]. Although there are many nonoperative management strategies to relieve the symptoms temporarily, discectomy and fusion are still the principal measure of treatment [5, 6]. However, spinal fusion changes the biomechanical properties of the spine and thus may accelerate adjacent segment degeneration [7].
Recent advances in synthetic materials and biological techniques have been adopted to simulate the architecture and function of the intervertebral disc (IVD) [8-16]. Since native interevertebral disc has a complex three-dimensional architecture and unique biological characteristics, novel strategies that are more effective and have fewer long-term side effects are needed.

It appears that regenerating degenerative organs could theoretically be one of the choices for overcoming the aforementioned hurdles. Decellularization is an attractive technique for preparing the specific organ or tissue extracellular matrix (ECM) scaffold. ECM could serve to regenerate organs and tissues [17]. Researchers use physical (e.g., shocks and freeze/thaw cycles), enzymatic (e.g., DNAase and trypsin) and chemical (e.g., 
Triton X-100 and sodium dodecyl sulfate (SDS)) protocols to decellularize organ or tissue of interest to acquire the organ-specific ECM [18]. For example, repeating freeze/ thaw cycles can disrupt cellular membranes and cause cell lysis, and chemical protocols can relatively mild effects upon tissue structure and solubilize both cytoplasmic and nuclear cellular membranes. Through removing allogenic or xenogenic cellular components, one could theoretically use these ECM to produce a minimally immunogenic scaffold with the proper biomechanical and biological properties. Indeed, several studies have tried to decellularization methods for regenerate IVD. Simionescu and $\mathrm{Xu}$ et al have decellularized the individual native-derived nucleus pulposus (NP) or annulus fibrous (AF) tissue [19-21]. Cheung et al [22] have attempted to decellularize a complete IVD, while only $70 \%$ of the endogenous cells were removed and complete decellularization was not achieved. Chan et al choose collagen type I as the main structure to obtain a cellderived decellularized NP [23]. However, there could be some problems because the major components of native NP ECM were collagen type II and glycosaminoglycans (GAGs). Here in our study, we try to decellularize the whole intervertebral disc completely and use this native ECM scaffold to treat disc degeneration.

\section{RESULTS}

\section{Optimized decellularization method preserved ECM components of IVD}

Different decellularization methods were tried before an optimal method was chosen for further study. After the whole IVD was decellularized using the optimal

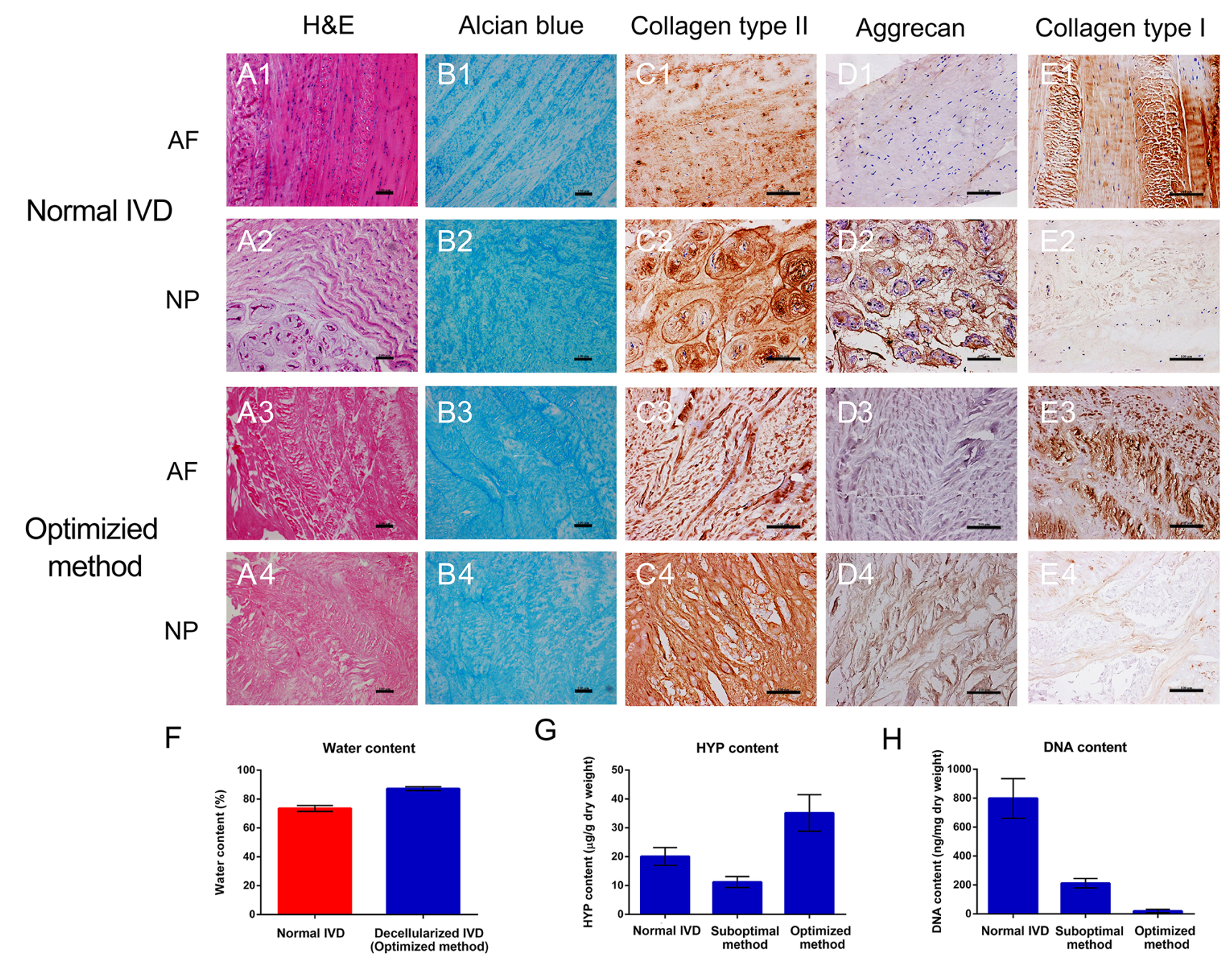

Figure 1: Optimized decellularization method preserved ECM components. H\&E, Alcian blue, collagen type II, AGN and collagen type I staining of normal A1.-2, B1.-2, C1.-2, D1.-2, E1.-2 and optimized method A3.-4, B3.-4, C3.-4, D3.-4, E3.-4. The content of DNA F., HYP G. and water H. revealed the excellent biological characteristics of optimized method group ( $n=5$ per study group). Optimized method includes $2 \%$ Triton X-100 for $24 \mathrm{~h}, 1 \% \mathrm{SDS}$ for $24 \mathrm{~h}$ and $200 \mathrm{U} / \mathrm{ml}$ DNAase for $12 \mathrm{~h}$. Abbreviations: IVD =intervertebral disc, $\mathrm{AF}=$ annulus fibrous, $\mathrm{NP}=$ nucleus pulposus, $\mathrm{HYP}=$ hydroxyproline. $\mathrm{A}-\mathrm{E}:$ Scale bar $100 \mu \mathrm{m}$. 
decellularization method, hematoxylin and eosin (H\&E) staining was performed to check the overall effect of the decellularization process on the removal of the cellular components. As shown in Figure 1A, 1H \& 1E staining of the decellularized IVD tissues showed pink eosinophilic staining that is typical of collagen, which was comparable with normal IVD. In contrast, no basophilic staining (indicative of cellular nuclear material) was detected in the decellularized tissues. Alcian blue staining (Figure 1B) revealed that the content of the GAGs is not reduced after decellularization. Moreover, comparable immunohistochemical staining revealed that the principal components of the IVD ECM, including collagen type II, aggrecan (AGN) and collagen type I, were remained well
Decellularized IVD (Optimized method)

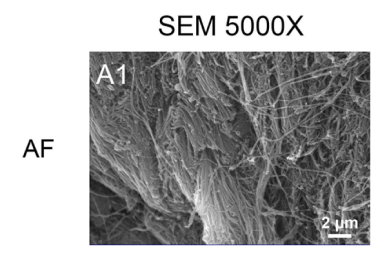

Normal IVD

\section{NP}
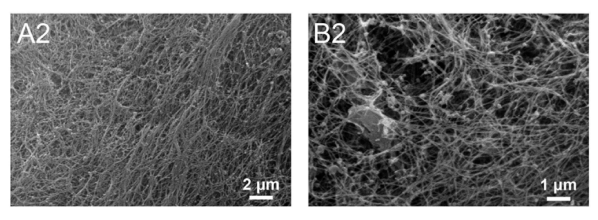

SEM $10000 X$
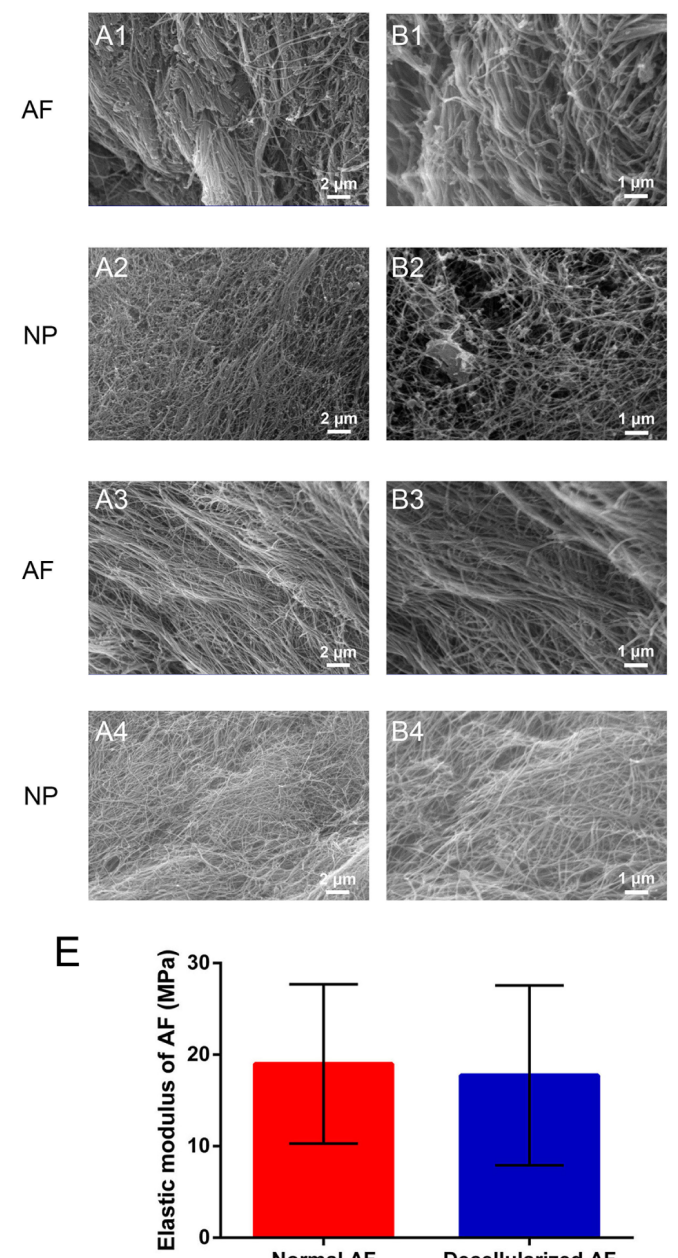

G
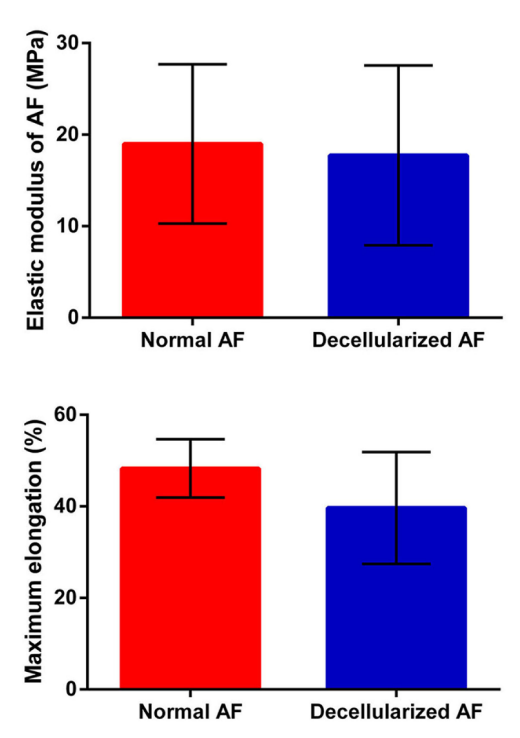

TEM 25000X
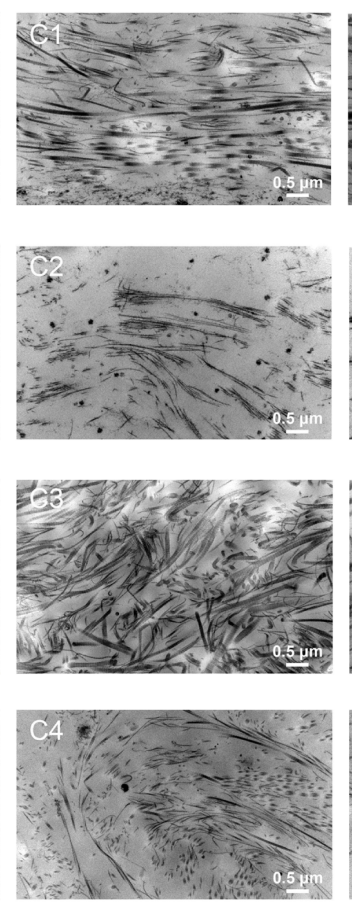

$\mathrm{F}$

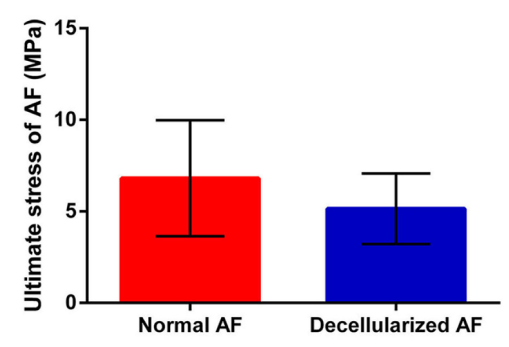

$\mathrm{H}$

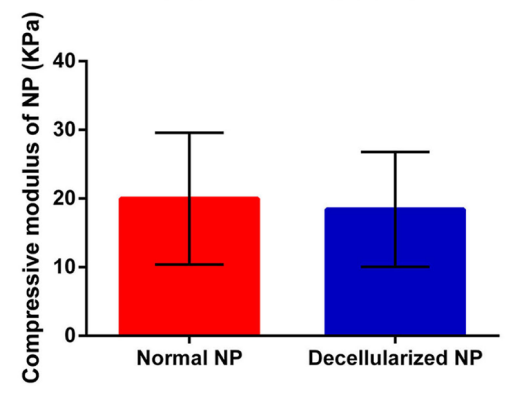

Figure 2: Optimized decellularization method preserved ECM structure and mechanical properties. Ultrastructure analysis reaveled that the collagen fibril meshwork in optimized method group A3.-4, B3.-4, C3.-4, D3.-4 is preserved and is similar to normal IVD A1.-2, B1.-2, C1.-2, D1.-2. The elastic modulus E., ultimate stress F. and maximum elongation G. indicate the mechanical change in AF after decellularization. The compressive modulus H. of normal NP and decellularized NP were measured at a $1 \mathrm{~Hz}$ loading frequency ( $n=6$ per study group). Abbreviations: IVD $=$ intervertebral disc, $\mathrm{AF}=$ annulus fibrous, $\mathrm{NP}=$ nucleus pulposus. 
in the optimized method group (Figure 1C, 1D and 1E). After decellularization process, the collagen type II and AGN also abound in NP and collagen type I still exists largely in AF. Interestingly the contents of hydroxyproline (HYP) and water were even increased in optimized method group $(20.05 \pm 3.09 \mu \mathrm{g} / \mathrm{g} v s .35 .15 \pm 6.35 \mu \mathrm{g} / \mathrm{g}$, $P=0.000 ; 73.52 \pm 2.03 \%$ vs. $87.22 \pm 1.34 \%, P=0.000)$ (Figure $1 \mathrm{~F}$ and $1 \mathrm{G}$ ). Furthermore, the degree of DNA removal in optimized method could reach up to $97.59 \pm$ $1.50 \%(P=0.000)$ (Figure $1 \mathrm{H})$. The destructive effect of suboptimal method on ECM components was evaluated and shown in Supplemental Figure 3. Therefore, optimized method using 2\% Triton X-100, 1\% SDS and $200 \mathrm{U} / \mathrm{ml}$ DNAase was chosen as the final decellularization protocol for further study.

\section{Optimized decellularization method preserved ECM structure and mechanical properties}

In addition completely removal of cells and the preservation of extracellular components, the preservation of ECM structure and mechanical property were also investigated. Scanning electron micrography (SEM) and transmission electron micrography (TEM) were applied to show the ultrastructural characteristics of ECM scaffold (Figure 2). SEM showed a well-organized parallel-aligned collagen fiber network in normal AF (Figure 2 A1 and 2

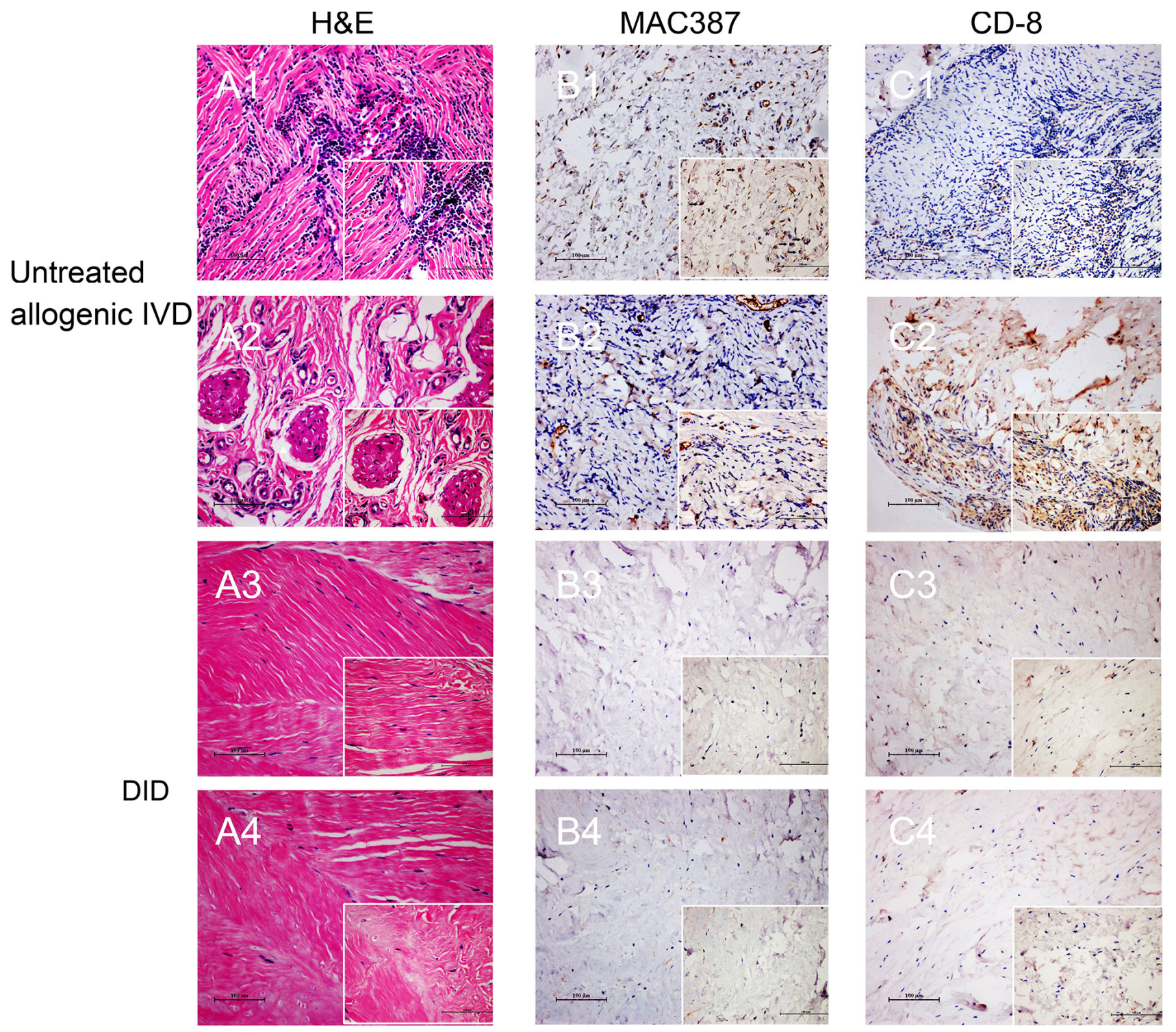

Figure 3: Decellularized allogenic IVD induced minimal immunological reaction in vivo. H\&E, MAC387 and CD-8 staining of untreated allogenic IVD A1.-2, B1.-2 and C1.-2 and decellularized allogenic IVD A3.-4, B3.-4 and C3.-4 IVD were performed after 1 month of subdermal implantation in rabbits. * Blood vessels, $\boldsymbol{\Delta}$ Foreign body granulomatous inflammation, $\rightarrow$ positive signal of MAC386 or CD-8. Abbreviations: IVD = intervertebral disc, DID = decellularized intervertebral disc. 
B1). In the decellularization group, the collagen fibers of the decellularized AF appeared to be more regular, and they also have a tiny and homogeneous space between the fibers compared with normal AF (Figure 2 A3 and 2 B3). The collagen of normal NP was intertwined and disorganized, which was similar to a complex meshwork that has fibers intercrossing between layers (Figure 2 A2 and 2 B2). The pores between the collagen fibers vary widely in normal NP. A similar pattern was found in the decellularized NP. The cross-cutting and mesh structure was well preserved after decellularization (Figure 2 A4 and 2 B4). The images of TEM further confirmed that the integration and continuity of the microstructure of IVD were well preserved after decellularization (Figure $2 \mathrm{C}$ and 2D). Together, these data suggested our decellularization methods well preserved the contents of ECM. In addition, the mechanical properties of decellularized IVD were retained well compared with normal IVD (Figure 2). The elastic modulus of annulus fibrous in decellularization group was similar to the than normal AF (17.74 $\pm 9.83 \mathrm{vs}$. $18.98 \pm 8.70 \mathrm{MPa}, P=0.821)$. The ultimate stress (UTS) and maximum elongation were also not obviously affected after decellularization (Figure $2 \mathrm{~F}$ and $2 \mathrm{G}, P=0.323$ and 0.202 , respectively). In addition, the compressive modulus of the decellularized NP was comparable to that of the normal NP $(18.41 \pm 8.38 v s .20 .00 \pm 9.59 \mathrm{kPa}, P=0.801)$ (Figure 2H). Collectively, the optimal decellularization methods preserved both ultrastructure and mechanical properties of the whole IVD.

\section{Decellularized allogenic IVD induced minimal immunological reaction in vivo}

As immunological response is critical for allogenic implant, the immunological response was first evaluated in vivo. All the animals survived, and no complications were observed during one-month implantation period. The histological evaluation indicated a high degree of host cell penetration into the native IVD, whereas only a few host cells were scattered in the decellularized IVD (Figure 3A). Neutrophils were observed extensively scattered around the grafts, which suggested a severe inflammatory response (Figure $3 \mathrm{~A} 1$ ). In addition, new blood vessels and classic foreign body granulomatous inflammation were found in the untreated allogenic IVD (Figure 3 A2). In contrast to the above inflammatory reaction, fewer inflammatory cells were observed, with only a small amount of fibroblasts dotted in the decellularization group (Figure 3 A3 and 3 A4). Immunostaining of the macrophages and CD8+ T-cells was used to determine the degree of cell-mediated immunoreaction. A large number of infiltrating CD8+ T-cells and macrophage cells were found in untreated allogenic IVD group (Figure 3 B1-2 and 3 C1-2). However, the percentage of positive cells of was fewer in decellularization group (Figure 3 B3-4 and 3 C3-4). Besides, the in vitro cytocompatibility of decellularized IVD was tested by cell counting kit-8 (CCK-8) and Live-Dead cell staining (Supplemental Figure 4) suggesting decellularized IVD has good cytocompatibility in vitro. Taken together, these results suggested that the decellularization process avoided a cellmediated immunoreactions to the grafts.

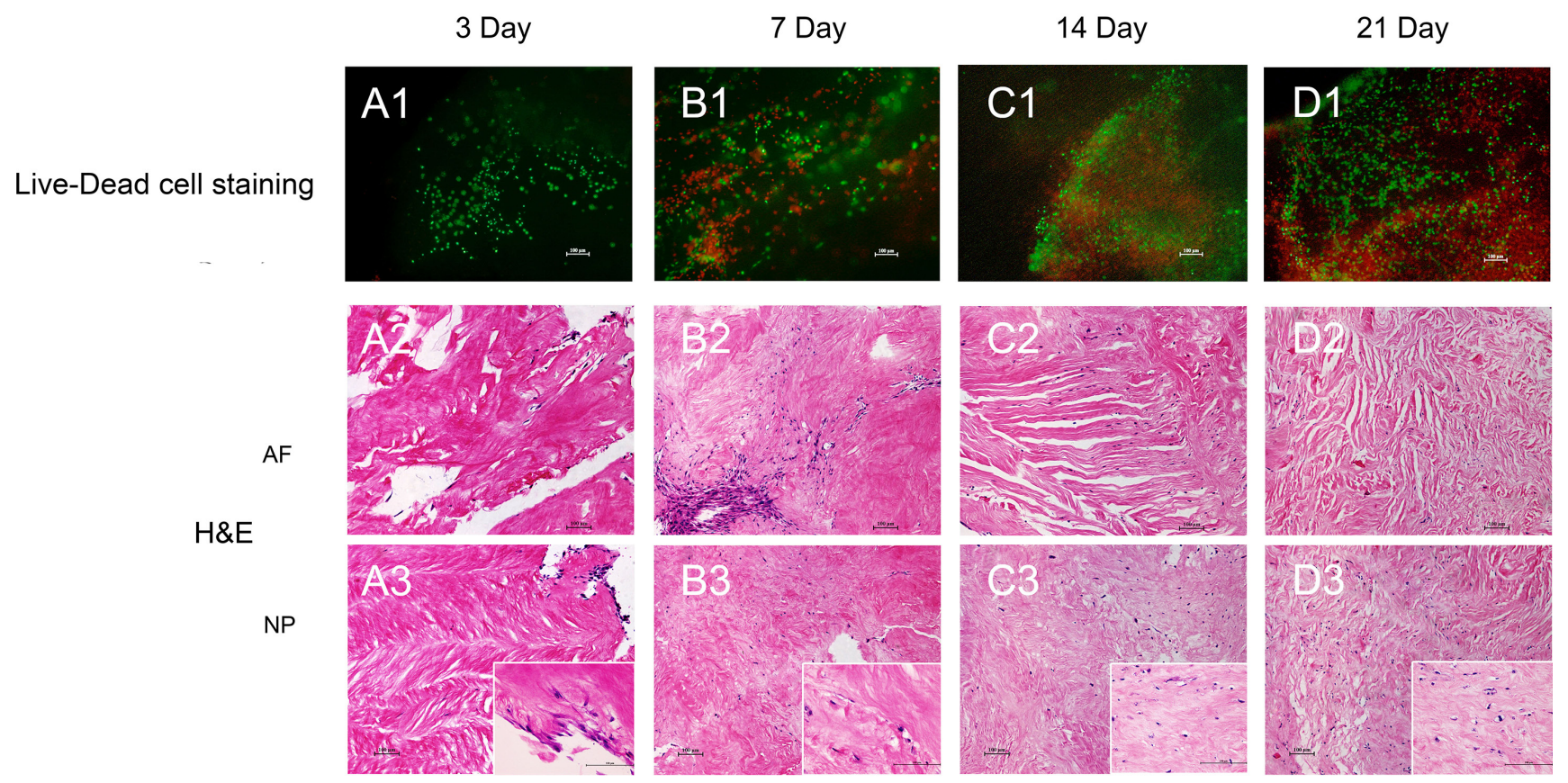

Figure 4: Decellularized ECM supports MSC proliferation in vitro. Live-Dead cell A1.-D1. and H.\&E. A2.-D2. and A3.-D3. staining of the MSC proliferation process. Abbreviations: AF = annulus fibrous, NP = nucleus pulposus. 


\section{Decellularized IVD ECM supports mesenchymal stem cell (MSC) proliferation in vitro}

For potential tissue regeneration application, the effect of decellularized IVD ECM on MSC was first evaluated in vitro, and before that, the native disc cells were confirmed completely removed after decellularization process (see above). Live-Dead cell staining indicated long-term viability of the MSCs after being seeded for 3, 7, 14 and 21 days (Figure 4). The live cells (green) showed that the majority of the seeded cells migrated into the inside of decellularized disc from day 3 to day 21 (Figure
4 A1-4 D1). The cells were predominantly found on the surface of the decellularized disc, with a small number of cells migrating into the inner region on day 3 (Figure 4 A1). As time progressed, more and more seeded cells proliferated and migrated into the decellularized disc (Figure 4 B1 and 4 C1). Even more intriguing, the seeded cells were distributed evenly throughout the decellularized disc on day 21 (Figure 4 D1). Furthermore, H\&E staining showed similar migration pattern. It is noticeable that the seeded cells are elongated and lined up along the collagen fibers in AF (Figure $4 \mathrm{C} 2$ ). More interestingly, the cell morphology changed from fusiform to round or oval at the site of the NP (Figure 4 C3 and 4 D2-3). 4, 6-diamidino-2-
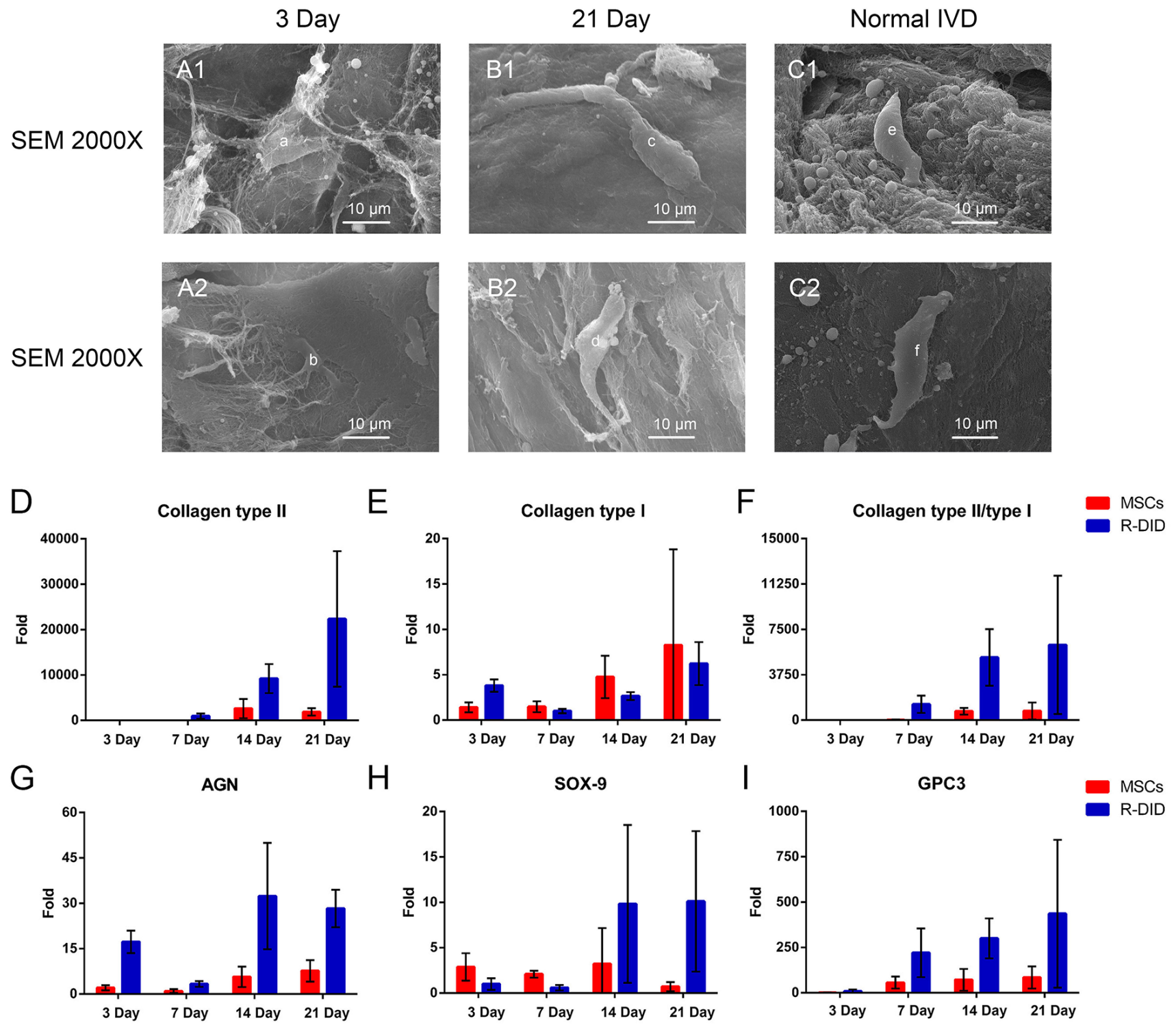

Figure 5: Decellularzied IVD ECM induced IVD-like cell differentiation in vitro. The dynamic changes in the cell morphologies A1.-2 and B1.-2 were observed, and normal IVD C1. and C2. was used as the reference. "a" and "b": Cells seeded into decellularzied IVD ECM on day 3; "c" and "d": Cells seeded into decellularzied IVD ECM on day 21; "e" and "f": Normal IVD cells. The classic IVDrelated gene expression of MSC seeded in ECM by RT-PCR D.-I.. Gene expression data are normalized to the average number. MSCs: Cells cultured in standard DMEM-HG medium ( $n=8$ per study group per time point). Abbreviations: IVD = intervertebral disc, R-DID $=$ recellularized decellularization $\mathrm{IVD}, \mathrm{AGN}=$ aggrecan . 
phenylindole (DAPI) staining was used to further confirm the MSCs proliferation in ECM (Supplemental Figure 5).
Additionally, cell metabolic activity and proliferation of MSCs were also assessed by the CCK-8 and DNA content

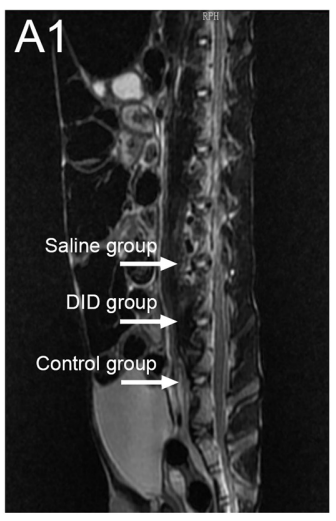

Pre-degeneration (0 month)

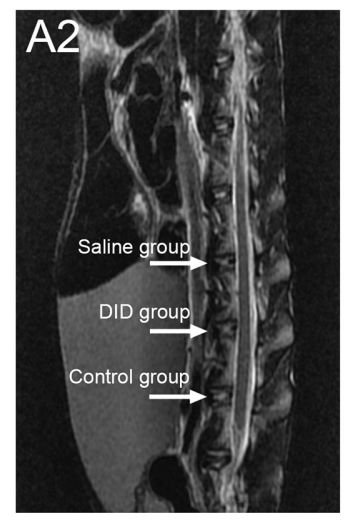

Pre-injection

(1 month)

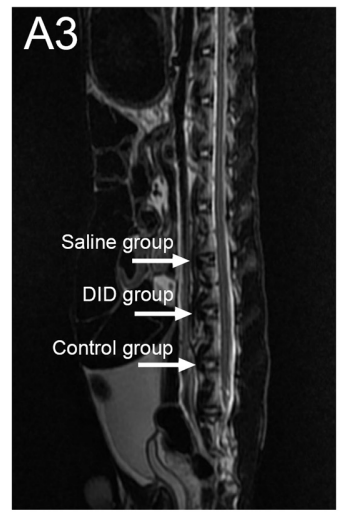

Post-injection (2 month)

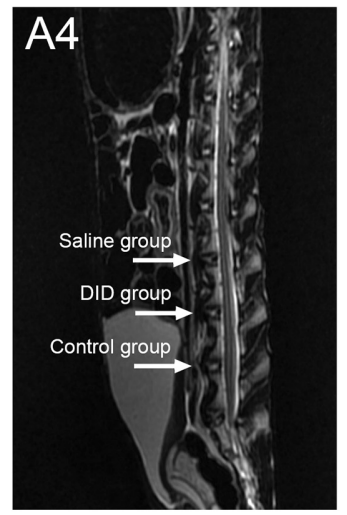

Post-injection (3 month)
B

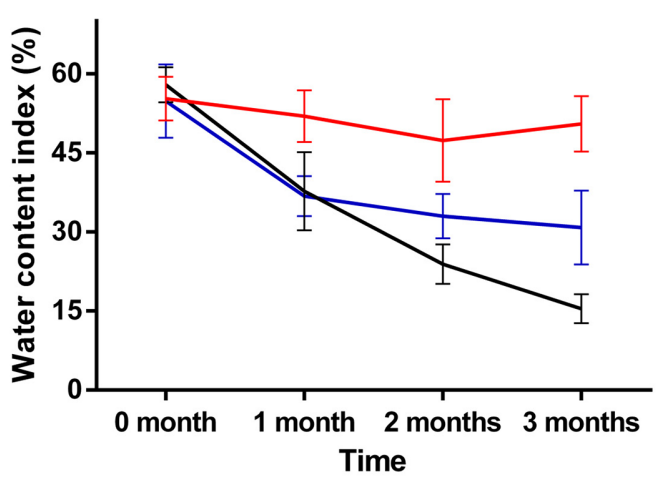

Control group
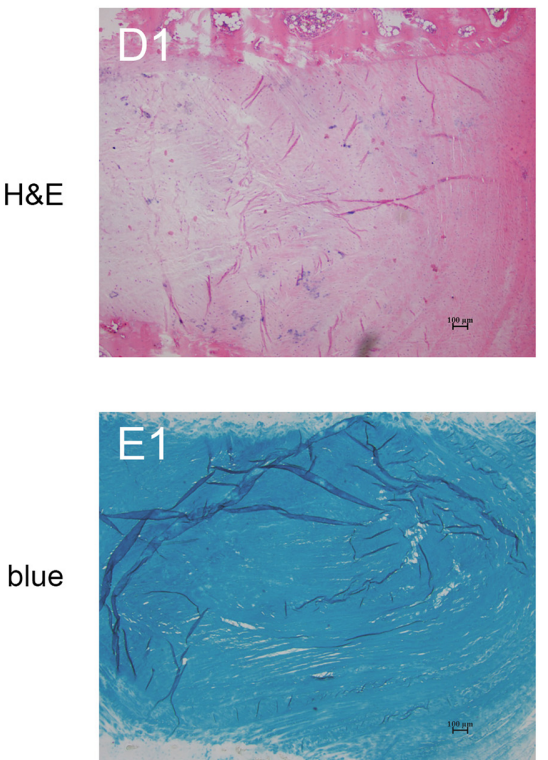

C

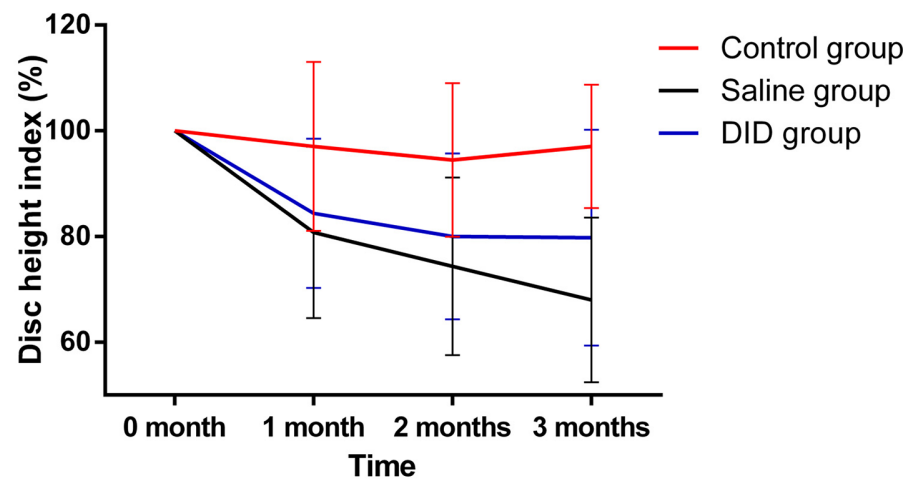

DID group

Saline group
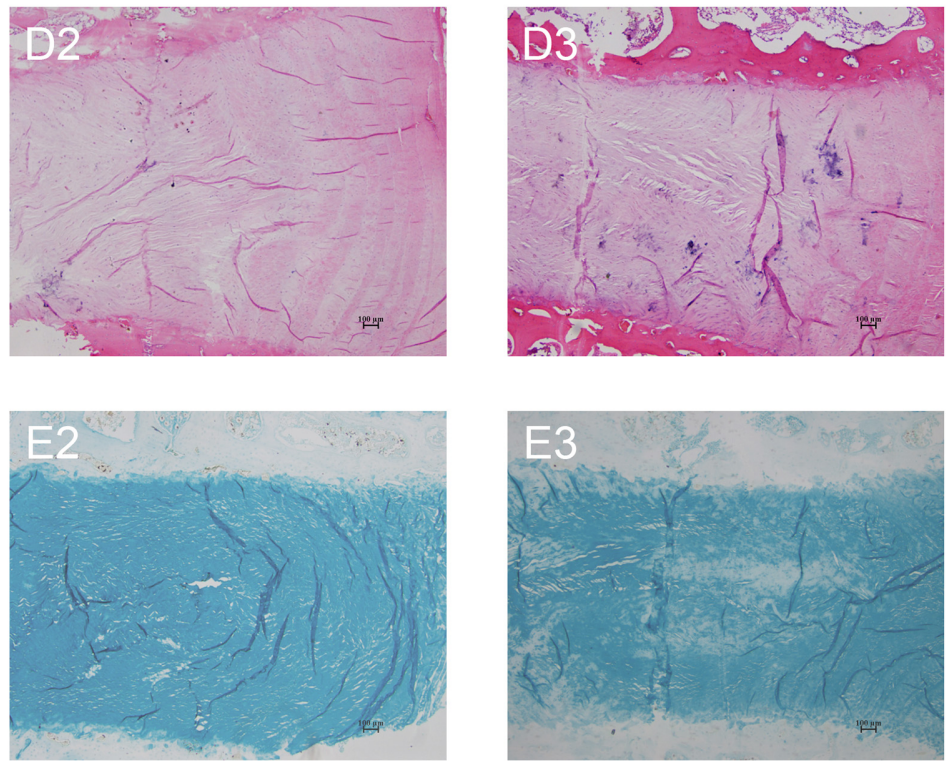

Figure 6: Decellularzied allogeneic IVD prevented disc degeneration in vivo. Continuous and dynamic changes of IVD magnetic resonance imaging (MRI) on T2-weighted images at different time points A.. Water content index B. and disc height index $\mathbf{C}$. indicated by MRI. H\&E and Alcian blue staining of normal (control), DID and saline IVDs at 2 months after injection ( $n=5$ per study group per time point). DID group: allogeneic IVD-derived ECM microparticles. Abbreviations: DID = decellularized IVD. 
assay, which were in agreement with the above results (Supplemental Figure 5).

\section{Decellularzied IVD ECM induced IVD-like cell differentiation in vitro}

Our histology result revealed morphological changes when MSCs cells are seeded on the decellularized IVD. Therefore, we further evaluated the morphology of these cells using SEM. SEM found IVD-like cell differentiation was induced with MSCs seeded in the decellularized IVD. The dynamic morphology changes were observed in Figure 5. At low magnification $(\times 2000)$, the thin and flat cell anchored onto the decellularized IVD surface, stretching with lengths that ranged to approximately $10 \mu \mathrm{m}$ on the three-day (Figure 5 A1-2). After 21 days of culturing, the cell body translated into a column or strip-like shape, and the cell shape was similar to normal intervertebral disc cells (Figure 5 B1-2 and 5 C1-2).

In addition to morphological observations, we compared the expression of IVD-related genes between cells that were seeded in decellularized IVD and nonseeded MSCs. Collagen type II is one of the most important IVD cell markers. The expression of collagen type II progressively increases during the culture period in the decellularization group. The expression pattern is consistently higher in the decellularization group than the control group, with an increase of more than 12-fold in the recellularized group on day 21 (Figure 5D). In contrast, no significant difference was found in the expression of collagen type I (Figure 5E). The expression of SOX-9, a classic IVD cell marker, was fourteen times $(P<0.05)$ greater than in the non-seeded MSCs in later stages (Figure $5 \mathrm{H})$. In agreement with this finding, the expression of the other IVD-cell markers, AGN and glypican 3 (GPC3), were significantly greater at different time points (Figure $5 \mathrm{G}$ and 5I). The novel IVD marker genes, forkhead box F1 (FOXF1) and carbonic anhydrase 12 (CA12) also showed large increases (118-fold and 5-fold, respectively, $P<0.05$ ) in the decellularization group (Supplemental Figure 6A and 6B). Two-way ANOVA showed that both time $(P<0.05)$ and group $(P<0.05)$ significantly affected the gene expression of AGN, GPC3, CA12 and FOXF1 and collagen type II/type I. Additionally, no significant changes were found in tissue inhibitor of metalloproteinase 1 (TIMP-1) and TIMP-2 expression (Supplemental Figure 6C and 6D). Transforming growth factor $\beta$ (TGF- $\beta$ ) families and their receptors, especially during the latter stages, were up-regulated in MSCs seeded decellularized IVD (Supplemental Figure 6E-6H). In all, these results suggested decellularized IVD supported IVDlike cell differentiation in vitro.

\section{Decellularzied allogeneic IVD prevented disc degeneration in vivo}

Given the potential of inducing MSC differentiate into IVD-like cell in vitro, we further evaluated the therapeutic effect of decellularized IVD in vivo. The effect of decellularzied allogeneic IVD microparticles was used to treat degenerative disc in rabbit. The continuous and dynamic changes of IVD in different groups at 0 month, 1 month, 2 months, and 3 months were demonstrated in Figure 6A. The water content index (Figure 6B) showed that decellularized IVD maintained a higher hydration level compared to the saline group, at both 2 months and 3 months $(32.96 \pm 4.24 \%$ vs. $23.90 \pm 3.77 \%, P=0.001$ and $30.82 \pm 7.00 \%$ vs. $15.45 \pm 2.76 \%, P=0.005$, respectively). Magnetic resonance imaging (MRI) showed that the disc height (Figure 6C) of the decellularized IVD treated group was also slightly better than the saline group, although a significant difference was not observed $(P=0.998$ and 0.281 , respectively). Figure $6 \mathrm{~B}$ and $6 \mathrm{C}$ indicated that the decrease of water content index and disc height was delayed to some extent in the decellularized IVD treated group. Moreover, in the decellularized IVD treated group, the histological morphology of collagen staining by H\&E did not show obvious changes compared with the control group. However, in the saline group, the inner layer of the AF lost the concentric lamellar structure with cracks, and the NP was also disorganized (Figure 6D). Alcian blue staining also shows that the obvious loss of GAGs in the saline group. But this loss was not apparent in the decellularized IVD treated group (Figure 6E). For future potential clinical applications, human-derived IDD cells (HDCs) were further seeded to investigate its therapeutic effect. Gene expression profiles of HDCs seeded in the decellularized IVD at day seven were measured. As shown in Supplemental Figure 7, the degeneration-specific markers, collagen type I and collagen type III expression were significantly decreased after being seeded in the decellularized IVD (72-fold and 2349-fold, respectively, $P<0.05$ ). However, the ratios of collagen type II to type I and type II to III were found to be significantly greater in the decellularized IVD group (9-fold and 16-fold, respectively, $P<0.05$ ). Additionally, FOXF1 expression tended to increase in the decellularized IVD group compared with the control group (3-fold, $P<0.05$ ). Collectively, these data suggested that decellularized IVD could prevent IVD degeneration.

\section{DISCUSSION}

Tissue regeneration is common choice for various end-stage diseases, and intervertebral disc degeneration is no exception. Here, this study reported an optimized decellularization method that could successfully obtain the IVD ECM scaffold without affecting its spatial framework, 
bioactivity and biomechanics. The decellularized allogeneic IVD induced minimal immunological reaction. By seeding MSCs into the ECM scaffold, we were surprised to find that IVD-like cell differentiation in vitro. Furthermore, this study confirmed the therapeutic effect of decellularzied allogeneic IVD on preventing disc degeneration in vivo.

IVD is a bulky and denser tissue. We applied physical treatments (snap freezing-thawing) before the chemical treatments (Triton X-100 and SDS) to disrupt cell membranes and release cellular components. To loosen the IVD tissue and thoroughly expose the cellular antigens to the decellularization reagents, a relatively long time and treatment with low concentration of decellularized reagents ( $2 \%$ Triton X-100 and 1\% SDS) has been proven effective. Our results showed that the main bioactivity components of IVD ECM (GAGs, AGN, collagen type II and collagen type I) were well preserved. It should be noted that the content of collagen and water have been 'increased' in the optimized decellularization protocol group. It is most likely because decellularization detergents could partially break up peptide bonds and expose more amino and carboxylic groups to form hydrogen bonding with water, which results in higher HYP and water content. A similar paradoxical phenomenon was also observed in other studies [23, 24]. Because insufficient removal of DNA fragments was associated with a greater risk of a pro-inflammatory or immune response and also had potential biosecurity problems [25, 26], we used DNAase to minimize the DNA content (less than 5\%). We well-controlled washing condition at a low temperature $\left(4^{\circ} \mathrm{C}\right)$ throughout the whole decellularization to reduce ECM ultrastructure damage (caused by proteases releasing from disrupted cells). And the SEM and TEM observations confirmed the good integration and continuity of our ECM microstructure. Besides, no significant decrease of mechanical properties (elastic and compressive modulus) after decellularization was further supporting the above finding. Therefore, based on the combined effect of the aforementioned decellularization procedures, we proposed an optimized decellularization protocol for rabbit intervertebral disc in this study.

For completely removing the cellular components and DNA fragments, we found the decellularized allogenic IVD induced minimal immunological reaction in vivo. The histological evaluation showed no obvious host inflammatory cells infiltration and classic foreign body granulomatous inflammation were found in the decellularized allogenic IVD. The degree of cell-mediated immunoreaction (macrophages and CD8+ T-cells) was kept at a low level in the decellularized IVD treated group. And some researchers believed the presence of residual cell remnants, including cellular antigens and DNA fragments, had negative impact on the newly seeded cells [27-29]. Thus, allogenic IVD using the optimal decellularzation method could provide scaffolds with little immunological reaction.

By seeding MSCs into the ECM scaffold, we were surprised to find that IVD-like cell differentiation was induced during the culture process via morphological observations and IVD-related genes analysis. The dynamic cell morphology changes (thin and flat to column and strip-like shape) from day 3 to day 21 indicated that the differentiation had occurred in the seeded cells. And we deduced that decellularzied ECM may induced IVD-like cell differentiation in vitro for the cell shape was similar to normal IVD cells. It is well confirmed by our IVDrelated gene expression results. The significant increases of classic IVD cell marker genes (collagen type II, AGN and SOX-9) and novel marker gene (GPC3) demonstrated that the differentiation of MSCs happened in the ECM is discogenic. For example, the expression of collagen type II (12-fold), AGN (8-fold) and SOX-9 (14-fold) were important for IVD cells homeostasis and usually applied to define the IVD phenotypes. The enhanced collagen type II to type I ratio (8-fold) indicates a discogenic trend in seeded cells rather than fibrogenic. In addition to that, recent studies have regarded GPC3 as a novel candidate IVD marker for distinguishing disc cells from articular chondrocytes [30, 31]. While the mechanism of IVD-like cell differentiation is not fully understood, we suspected that the decellularized ECM may play an important role on it. The well-preserved bioactivity proteins (collagen type II, AGN, collagen type I, and GAGs) and intrinsic microstructures could possible provide the protein 'footprints' of the previous resident cells (normal intervertebral disc cells) to guide cell proliferation and differentiation. TGF- $\beta$ families (secreted factors) and their receptors were associated with MSCs differentiating to IVD cells and had therapeutic effect on disc degeneration by increasing collagen type II and AGN [12, 32-34]. Their high expressions during the latter stages may also had related to the cell differentiation.

The IVD-cell differentiation mechanism was needed to be explored in the following study but we confirmed the therapeutic effect of decellularzied allogeneic IVD on preventing disc degeneration in vivo. In the field of disc degeneration, growth factor supplementation and MSC-based therapies have been investigated by many researchers [23, 35-37]. Transplanting MSCs into collagen sponge, actelocollagen and hyaluronan gel to repair disc degeneration (improving the ECM, water content and disc height index (DHI)) has been proven to be effective [38-40]. Recently, Borde B et al and Grunert $\mathrm{P}$ et al proposed that high-density collagen is capable of repairing AF defects (induced by needle puncture) [41, 42]. However, there are few studies that have focused on the effect of decellularzied IVD ECM on treating disc degeneration. The outcomes (MRI and histology) of our animal degeneration experiment demonstrated that the injection of decellularized allogeneic ECM microparticles into degenerated disc can improve the degeneration and 
delay the degeneration process by increasing the water content, disc height, and ECM integrity and content. The regenerating effect of ECM microparticles could be attributed to the native microenvironments, and the active ingredients of ECM were preserved after decellularization. The role of decellularzied allogeneic IVD in preventing disc degeneration in vivo may be consistent with the effect on promoting MSC proliferation and differentiation and improving the HDCs phenotypes. Additionally, upon decellularization, the peptide bonds were partially broken up and exposed more amino and carboxylic groups to form hydrogen bonding with water; the resulting ECM scaffold has favorable hydrophilic properties and higher water content in the degenerating disc. Moreover, according to previous research results, high-density decellularized ECM microparticles (which mainly contain collagens and GAGs) could have a positive effect on the AF defect repairs in the injured intervertebral discs [41, 42].

There were several limitations in the present study. Although differentiating MSCs to IVD-like cells using decellularized scaffolds has been successful, other possible approaches are worthy of being pursued. First, different culture conditions, including hypoxia, non-contact co-culture or contact co-culture with IVD cells and supplementation of growth factors, should be further explored. Second, because of the lack of definite IVD cell markers, the specific markers are needed to confirm the differentiation of MSC toward IVD-like cells. Future research directions will focus on establishing the orthotopic whole IVD implantation model and using proteomic approaches to analyse the composition of IVD ECM after decellularization.

\section{CONCLUSIONS}

The current study showed an optimized decellularization method to completely remove the cellular components of the IVD while maintaining a threedimensional architecture, biochemical characteristics and biomechanical properties. Good immune tolerance and no cytotoxicity of our ECM scaffold were demonstrated. Interestingly, MSCs cultured in the decellularized IVD ECM scaffold were observed to differentiate to IVD-like cells. Moreover, the effects of decellularized IVD allogeneic ECM microparticles on treating disc degeneration and delaying the process of degeneration were revealed. Thus, this study showed that the allogeneic decellularized IVD ECM scaffold has therapeutic potential for treating disc degeneration.

\section{MATERIALS AND METHODS}

\section{Research overview}

The research framework was depicted in Supplemental Figure 1. IVD samples were harvested from rabbits. The cellular components were completely removed but the structure of ECM was well preserved. The determination of an ideal decellularization protocol was based on histological, DNA and collagen content analyses. Additionally, the mechanical properties of decellularized IVD were evaluated by testing the elastic and compressive modulus. To assess the biological safety of allogenic decellularized IVD, it was implanted into allogeneic subcutaneous tissue in vivo. MSCs were seeded in decellularized IVD, and the effect of ECM scaffold on MSCs proliferation and differentiation was evaluated using histological, biochemical, and real-time PCR (RTPCR) methods and SEM. Moreover, decellularized IVDderived allogenic ECM microparticles were injected in vivo to investigate the effect on preventing disc degeneration. Besides, HDCs were also isolated and reseeded into decellularized IVD to observe the effect of decellularized IVD on HDCs degeneration in vitro.

\section{Harvesting of rabbit IVD}

IVD samples were collected from the thoracic and lumbar segments of New Zealand White rabbits that were aged approximately four months old ( $n=55$, of which 45 were used for the in vitro study and ten for in vivo research). Muscle tissues and bone chips were carefully removed from each sample. The cartilage endplates were resected, then lavaged the IVD (including AF and NP) with phosphate-buffered saline (PBS) to eliminate the excess blood. The harvested IVDs were immediately frozen in liquid nitrogen for further use. IVD for DNA and HYP analysis must be freeze-dried via ice sublimation under a high vacuum in a vacuum freeze dryer (Marin Christ, Osterode, Germany) to normalize the specimens.

\section{Decellularization of IVD}

An optimized decellularized protocol was necessary to completely remove the cellular components of IVD and maximally retained the complicated threedimensional structure and biomechanics of the native ECM. To facilitate the diffusion of the cellular content and decellularized reagents, snap freezing-thawing cycles were used prior. Harvested IVDs were placed in a $37^{\circ} \mathrm{C}$ water bath and liquid nitrogen in rapidly alternating treatments for five cycles. First, 2\% Triton X-100 (Sigma) in deionized water was applied to soak the IVDs for $24 \mathrm{~h}$ 
on an orbital shaker at $100 \mathrm{rpm}$ and at $4^{\circ} \mathrm{C}$. Second, $1 \%$ SDS (Sigma) in deionized water was applied for $24 \mathrm{~h}$ to further remove the nuclear remnants and cytoplasmic proteins under the above conditions. The complete removal of DNA remnants from the tissue was fairly difficult due to their 'sticky' nature, which caused them to easily adhere to ECM proteins. To minimize the DNA content in the ECM, $200 \mathrm{U} / \mathrm{ml}$ DNAase (Sigma) in PBS was applied with shaking at $100 \mathrm{rpm}$ and at $37^{\circ} \mathrm{C}$ for $12 \mathrm{~h}$. Finally, the IVDs were rinsed with PBS for $6 \mathrm{~h}$ to remove the residue reagents effectively. Before establishing the above optimized decellularization protocol, we explored a suboptimal preparative method in which the concentrations of Triton X-100 and SDS in deionized water were $3 \%$ and $2 \%$, respectively, and no DNAase was incorporated. By applying the optimized decellularization protocol to IVD, we found that decreasing appropriately the concentration of decellularized agents (Triton X-100 and SDS) will not affect the removal rate of cells and can obvious reduce the damage of ECM. Besides, DNAase can minimize the DNA content and lower risk of a proinflammatory or immune response.

\section{Isolation and culture of rabbit MSCs}

Rabbit MSCs were isolated from the bone marrow of four-month-old New Zealand White rabbits $(n=5)$ by gradient isolation of mononuclear cells, and they were cultured in Dulbecco's modified Eagle medium with high glucose (DMEM-HG) (Gibco) plus 10\% fetal bovine serum (FBS) (Gibco), $100 \mathrm{U} / \mathrm{mL}$ penicillin, $100 \mu \mathrm{g} / \mathrm{mL}$ streptomycin, and $2.5 \mu \mathrm{g} / \mathrm{mL}$ amphotericin $\mathrm{B}$ at $37^{\circ} \mathrm{C}$ in a humidified atmosphere that contained $5 \% \mathrm{CO}_{2}$. Cells were plated in T-75 flasks and were allowed to adhere for $24 \mathrm{~h}$. Non-adherent cells were removed by three washes with PBS, and the adherent cells were cultured in complete DMEM-HG. Cells were detached using $0.25 \%$ trypsin-EDTA upon $80-90 \%$ confluence and were subcultured as passage one (P1). Cells at P3 were used for the recellularization of the decellularized IVD.

\section{Isolation and culture of HDCs}

HDCs were collected from five patients with IDD caused by lumbar disc herniation, who underwent discectomy and fusion in the Department of Orthopaedics, Sir Run Run Shaw Affiliated Hospital of Zhejiang University School of Medicine. Informed consent was obtained from each patient, and the research protocol was approved by the Ethics Committee of the Sir Run Run Shaw Affiliated Hospital of Zhejiang University School of Medicine. Bone chips and excess blood were carefully removed from each sample using surgical blades and forceps. The human IVDs were dissected into small pieces using a scissor. The tissues were then digested using $0.2 \%$ collagenase solution (Sigma) in standard DMEM for $8 \mathrm{~h}$ at $37^{\circ} \mathrm{C}$ with vibration. After digestion, the debris was filtered out with a cell strainer, and the cells were centrifuged at $800 \mathrm{rpm}$ for $5 \mathrm{~min}$. The HDCs were then cultured in T-75 culture flasks at $37^{\circ} \mathrm{C}$ in a $5 \% \mathrm{CO}_{2}$ atmosphere. The cells were also detached using $0.25 \%$ trypsin-EDTA upon 80 $90 \%$ confluence, and they were subcultured as passage $\mathrm{P} 1$. The cells at P2 were used for implanting into the decellularized IVD.

\section{Seeding MSCs into the IVD ECM scaffold}

To test the effect of decellularized IVD on MSCs proliferation and differentiation, the ECM samples were incubated in DMEM-HG medium for $12 \mathrm{~h}$ at $37^{\circ} \mathrm{C}$. A few studies proposed that seeding the cells several times was superior to seeding a single time for the cell viability and distribution in ECM $[27,44]$. In this study, a concentration of approximately $7 \times 10^{6}$ cells $/ \mathrm{mL}$ rabbit MSCs were introduced at each step, for a total of three steps, with 1 $\mathrm{h}$ intervals between each step. After $1 \mathrm{~h}$ of finishing the third seeding to allow sufficient adhesion to the scaffold, DMEM-HG medium was then carefully supplemented to a 24-well plate that contained recellularized scaffold (Supplemental Figure 1 and Supplemental Figure 2). The same source of MSCs was seeded in a Petri dish with a concentration of $5 \times 10^{5}$ cell $/ \mathrm{mL}$ and used as the negative group.

\section{Seeding HDCs into the IVD ECM scaffold}

It is believed that ECM can delay replicative senescent chondrocyte (which is similar to an IDD cell) dedifferentiation and enhance redifferentiation [45]. We therefore sought to seed $7 \times 10^{6}$ cell $/ \mathrm{mL}$ HDCs into decellularized IVD to evaluate the improved effect on degenerative cells. Cells from the same source were seeded in a Petri dish at a concentration of $5 \times 10^{5}$ cell $/ \mathrm{mL}$ and used as the corresponding negative control group.

\section{Rabbit Subcutaneous implantation of decellularized IVD}

For allograft cellular components can lead to immune reaction and affect the treatment effect, so allograft transplant was used to test the immunogenicity of decellularized IVD. New Zealand White rabbits $(n=5)$ were utilized for the study. Each rabbit was anesthetized with an intraperitoneal injection of chloral hydrate $(5 \mathrm{ml} /$ $\mathrm{kg}$ ). Three subdermal pockets were made on either side of the dorsal midline of each rabbit by making small incisions $(1 \mathrm{~cm})$. Decellularized IVDs $(n=3)$ and normal IVDs $(n=3)$ were separately implanted into corresponding pockets through the incision. Incisions were closed with 
surgical ligation. One month after implantation, implants were removed and prepared for histological evaluation by submersion in 4\% PBS-buffered paraformaldehyde (PFA).

\section{Evaluation of histological, histochemical, immunohistochemical and fluorescent staining}

The normal IVD, decellularized IVD, subcutisembedded specimens and recellularized decellularization IVD at 3, 7, 14 and 21 days were washed three times with PBS and fixed in 4\% PFA for $24 \mathrm{~h}$, followed by standard paraffin embedding and sectioning. For treatment of IDD study, IVD samples were harvested from rabbits at 2 months after injection of decellularized IVD-derived ECM microparticles, fixed in 4\% PFA for $48 \mathrm{~h}$ and decalcified for at least 30 days before embedding in paraffin wax. After sectioning, routine H\&E staining was performed to reveal the complete distribution of various cells and cells with different morphologies. Alcian blue staining was used to identify the GAG content that was retained in decellularized IVD, recellularized decellularization IVD and IVD samples. Specimens for immunohistochemistry were possessed as previously described by our group [46]. Epitopes of interest included collagen type II (Novus Biologicals, Littleton, USA), AGN (Novus Biologicals, Littleton, USA), collagen type I (Abcam, Cambridge, US), CD8 (Novus Biologicals, Littleton, USA) and MAC387 (Abcam, Cambridge, US). The hematoxylin and Vectastain ABC kit (Vector Laboratories, Burlingame, USA) was used for visualization of the cell nuclei and the positive signal in the specimens, respectively. Specimens that were incubated for $5 \mathrm{~min}$ in DAPI (Sigma) fluorescent nuclei stain were used to observe the proliferation of the seeded cells in decellularized IVD at different time points.

\section{Determination of DNA, collagen and water content}

The content of total genomic DNA of normal IVD, decellularized IVD, and recellularized decellularization IVD at different time points was extracted and isolated using a DNeasy Blood \& Tissue Kit (Qiagen, Hilden, Germany) in accordance with the manufacturer's protocol and was previously described by our group [47]. The DNA content was measured with a NanoDrop 8000 (Thermo Fisher Scientific, Wilmington, USA). As a marker amino acid of collagen, the content of HYP can be measured using a spectrophotometric method [48]. The amount of HYP in the samples was determined by a calibration curve with a linear region between 0.2 and $1 \mathrm{mg} / 100 \mathrm{~mL}$, prepared from HYP standards (Sigma). The mass changes of normal and decellularized IVDs after freeze-drying were calculated to evaluate the water content.
Scanning and transmission electron microscopy

SEM and TEM were performed to examine the micro-architecture of the normal and decellularized IVD. $\mathrm{NP}$ and AF tissue samples were dissected out and fixed in $2.5 \%$ glutaraldehyde in PBS overnight at $4{ }^{\circ} \mathrm{C}$, followed by three washes in PBS. The fixed samples were post-fixed with $1 \% \mathrm{OsO} 4$ for $1 \mathrm{~h}$ and washed with PBS three times, as above. The samples were then dehydrated in a graded series of ethanol and dried in a critical point dryer (HCP-2, Hitachi, Japan) with liquid $\mathrm{CO}_{2}$. Subsequently, the dried samples were sputter-coated with gold-palladium and viewed under the SEM (EM-3200, KYKY, China).

For the TEM, we fixed and post-fixed AF and NP samples as above. The samples were infiltrated in a 1:1 mixture of absolute acetone and resin for $1 \mathrm{~h}$, and then, they were transferred to a 1:3 mixture of absolute acetone and the final resin for $3 \mathrm{~h}$ and, then, transferred to $100 \%$ resin overnight. The samples were placed in capsules that contained embedding medium and were heated at $70^{\circ} \mathrm{C}$ for $9 \mathrm{~h}$. The hardened blocks were ultra-thin sectioned at $70 \mathrm{~nm}$ with a diamond knife using an ultramicrotome (EM UC7, Leica, Germany) and placed on copper grids. Then, the specimen sections were stained by uranyl acetate and alkaline lead citrate for $15 \mathrm{~min}$ and observed by a TEM (H-7650, Hitachi, Japan).

\section{Cytocompatibility testing of decellularized IVD}

The decellularized IVDs were incubated in DMEMHG medium for $48 \mathrm{~h}$ in a humidified atmosphere with 5\% $\mathrm{CO}_{2}$ at $37^{\circ} \mathrm{C}$, and the medium was collected for later use (leaching solution). The leaching solution was generated by each decellularized IVD incubated in the $1 \mathrm{ml}$ DMEMHG medium. MSCs at P2 were seeded in 96-well cell culture plates in $200 \mu 1$ standard DMEM-HG medium at a concentration of $5 \times 10^{3}$ cells/well for $24 \mathrm{~h}$. The medium was then removed and replaced with the leaching solution $(25 \%, 50 \%$ and $100 \%)$ and incubated for six days. CCK8 was added to each well of the plate, and the plate was incubated for $3 \mathrm{~h}$ in the incubator at the time points of one, two, three, four, five and six days. The Live-Dead cell staining (BioVision, Milpitas, USA) was used to distinguish between live and dead cells and observe the overview of the situation of cellular activity. The detailed description of CCK-8 and Live-Dead cell staining process can be obtained in our previous studies [47, 49]. The stained live (green) MSCs at the above time points can be visualized by fluorescence microscopy. The metabolic activity of MSCs was detected by CCK- 8 and compared with the positive group (cells cultured in standard DMEMHG medium) to determine the cytocompatibility of the decellularized IVD samples. 


\section{Biomechanical testing}

The mechanical properties of AF and NP after decellularization were measured using uniaxial tensile and dynamic strain tests, which performed using a computercontrolled test machine (Z2.5, Zwick/Roell, Germany) and had been described and validated by our previous studies [50-52]. For AF, the samples were clamped to the grips in the mechanical apparatus, and the initial specimen's width, length and thickness were recorded. The samples were then stretched to tensile failure at a rate of $10 \mathrm{~mm} /$ min. The stress-strain curves were collected. The UTS was calculated by dividing the maximum load by the crosssectional area of the specimen. The maximum elongation was calculated by dividing the strain at a failure length by the initial length of the specimen. The elastic modulus was calculated from the slope of the ascending linear region of the stress-strain curve. For NP, the samples were maintained in $37^{\circ} \mathrm{C}$ PBS until testing. The samples were mounted into the specific round hole $(4.6 \mathrm{~mm}$ in diameter and $3 \mathrm{~mm}$ deep), and the top loading shaft was then slowly loaded to the NP. The oscillatory amplitude was set at 0.3 $\mathrm{mm}$, which generated a dynamic strain level of $10 \%$. The dynamic strain was applied at the loading frequencies of $1 \mathrm{~Hz}$, which was similar to physiological conditions. The compressive modulus of $\mathrm{NP}$ at $1 \mathrm{~Hz}$ was calculated after the whole test cycle from the slope of the ascending linear region of the stress-strain curve.

\section{Cell viability and metabolic activity of the recellularized decellularization IVD}

The viability of cells in the recellularized decellularization IVD was determined by Live-Dead cell staining. The samples were incubated with calcein acetoxymethyl ester and ethidiumhomodimer- 1 for $20 \mathrm{~min}$ at $37^{\circ} \mathrm{C}$ in darkness, to simultaneously but separately stain both the living and dead cells. After washing with PBS to remove the labeling reagents, the stained samples were visualized by fluorescence microscopy. The metabolic activity of the recellularized decellularization IVD cells was assessed by CCK-8, and the incubating time was adjusted to be $2 \mathrm{~h}$. The corresponding absorbance was transformed into cell numbers by using the standard curve.

\subsection{Gene expression of IVD cell markers by RT- PCR}

Because the ECM proteins which newly deposited by recellularized decellularization IVD cells was difficult to distinguish from the ECM that remained after decellularization, gene expression rather than protein expression of a panel of IVD cell markers was evaluated. RNA from MSCs-seeded decellularized IVD, HDCs- seeded decellularized IVD and monolayer controls was isolated using RNeasy Mini kit (Qiagen, Valencia, CA, USA) according to the instructions [49]. RNA integrity and quantification was detected by using NanoDrop 8000. It was then reverse transcribed using $1 \mu \mathrm{g}$ of RNA from each sample, $2 \mu$ of $5 \times$ PrimeScript RT Master Mix (Takara Bio, Otsu, Japan), and $4 \mu \mathrm{l}$ of RNase Free dH2O in a total volume of $10 \mu \mathrm{L}$. After amplifying the cDNA, RT-PCR was performed on a 96-well plate ABI Prism 7500 (Applied Biosystems, Foster City, CA, USA) using SsoFast EvaGreen supermix (Bio-Rad, Hercules, CA, USA). The total volume $(20 \mu \mathrm{L})$ of each PCR reaction contained $10 \mu \mathrm{L}$ of SsoFast EvaGreen supermix, $7 \mu \mathrm{L}$ $\mathrm{ddH} 2 \mathrm{O}, 2 \mu \mathrm{L}$ cDNA and $10 \mu \mathrm{M}$ of each of the forward and reverse primers (Supplemental table 1). The expression levels of thirteen genes (collagen type II, collagen type I, SOX-9, GPC3, FOXF1, AGN, CA12, TIMP-1, TIMP2 , TGF- $\beta$ families and their receptors) were chosen to investigate the progress of IVD regeneration and its possible mechanism. In addition, the expression levels of collagen type II, collagen type I, collagen type III and FOFX1 in HDCs seeded decellularized IVDs were used to evaluate whether decellularized IVD has effect on degenerative cells. The expression ratio for each marker was quantified mathematically by the $2^{-\Delta \Delta C t}$ method using $\beta$-actin or 18S rRNA as the housekeeping gene, and the target genes were compared relative to the cells cultured in standard DMEM-HG medium.

\section{Establishment of the rabbit IVD degeneration model}

IVD degeneration was induced by the validated rabbit puncture model [53]. This puncture model initiated a reliable cascade of MRI and histologic changes that resembled the hallmarks of human IVD degeneration (darkening and collapse of disc spaces). Prior to the procedure, the animals were put under general anesthesia. The rabbit's spine was exposed from an anterolateral retroperitoneal approach with aseptic techniques. L4L5 and L3-L4 IVDs were sequentially punctured with a 22-gauge needle to a depth of $5 \mathrm{~mm}$, and then, the surgical incisions were closed. L5-L6 was left uninjured as the normal control. The rabbits were allowed to move freely around their cages.

\section{Injection of decellularized IVD-derived ECM microparticles}

After cutting the decellularized IVD samples into pieces, an appropriate amount of sterile saline was adding, and then, tissue homogenizer (Bertin * Precellys 24, Bertin Technologies) was applied to grind the samples at $6000 \mathrm{rpm}$ for 30 seconds for approximately 20 circles. The temperature during homogenization should remain at $4^{\circ} \mathrm{C}$ 
to prevent protein denaturation. After homogenization, the suspension was obtained and screened through the 80 -mesh sample sieve $(0.2 \mathrm{~mm})$. The size of the decellularized IVD-derived ECM microparticles (suspension of homogenised ECM) was controlled to be less than $200 \mu \mathrm{m}$. The concentration of the DID-derived ECM microparticles suspension was $50 \mathrm{mg} / \mathrm{ml}$.

Injection of the decellularized IVD-derived ECM microparticles was performed one month after degeneration induction. L5-L6 was used as the control group. L4-L5 and L3-L4 were assigned to two groups, namely, the decellularized IVD group and saline group, respectively. Under general anaesthesia and guided by X-rays, L5-L6, L4-L5, and L3-L4 IVDs were exposed from the other side (contralateral to the previous puncture surgery). L4-L5 and L3-L4 IVDs were injected with a 50 $\mu 1$ suspension of ECM microparticles and a $50 \mu 1$ sterile saline through a Hamilton syringe with a 27-gauge needle, respectively. The small needle size was selected to avoid exacerbating further degeneration.

\section{MRI evaluation}

MRI was performed with a 1.5-Tsystem (GE Signa Excite; GE Healthcare, Milwaukee, Wisconsin) on the rabbits at 0 month, 1 month, 2 months and 3 months to indicate the water content and heights of the discs. Rabbits were placed supine, and the human lumbar sequences with a knee-joint surface coil were used. First, T2-weighted coronal scouting images were acquired at the lumbar spine, and the image with the most intervertebral discs was selected. Then, T2-weighted sagittal scouting images were acquired according to the previously selected coronal locating image. Additionally, a central sagittal image of the lumbar spine was selected as a locating image for the next cross-sectional scans. The middle images for each of the IVD locations at L5-L6, L4-L5, and L3-L4 were selected by locating lines on sagittal plane MRI scans. The window width and window level were 3600 and 1800 for fat-suppressed T2-weighted images, and detailed manipulation were previously described by our group [54].

Measurements were made with the software of ImageJ 1.46 (National Institutes of Health, http://rsbweb. nih.gov/ij/download.html). The mean signal intensity values of T2-weighted imaging of IVD and cerebrospinal fluid in the central sagittal plane were measured, and the ratio between them was calculated to reflect the water content of each IVD. The height of the IVD was also measured and was expressed as the DHI. The average measurement of the anterior, middle and posterior height of the disc was divided by the standard calibration line of each image. The change in the DHI was expressed as the percentage DHI $(\% \mathrm{DHI})$ and normalized to the DHI that was obtained from the pre-degeneration (0 month) T2-weighted imaging. The above measurements were performed by 3 people ( 2 radiologists and 1 spine surgeon) independently, and the results were averaged.

\section{Statistical analysis}

Quantitative results are represented as a mean \pm standard deviation. One-way ANOVA or two-way ANOVA was performed to detect the differences in the content of DNA, HYP and water, the mechanical properties, expression levels of the genes, IVD functional outcomes and cytocompatibility among different treatment groups. Statistical analysis was performed using SPSS 19.0 software (SPSS Inc, Chicago, USA), and significance was defined as $P<0.05$.

\section{CONFLICTS OF INTEREST}

Xianfeng Lin, Xiangqian Fang, Qiang Wang, Zhijun $\mathrm{Hu}$, Kai Chen, Zhi Shan, Shuai Chen, Jiying Wang, Jian Mo, Jianjun Ma, Wenbing $\mathrm{Xu}$, An Qin, Shunwu Fan declare that they have no conflict of interest

\section{GRANT SUPPORT}

The authors declare that they do not have anything to disclose regarding funding from industries with respect to this manuscript. This work was supported by National Natural Science Fund of China (81472064, 81301585 and 81171739), Zhejiang Provincial Natural Science Foundation of China (LQ13H060001), and Zhejiang medical and health science and technology project (2013KYA112).

\section{Editorial note}

This paper has been accepted based in part on peerreview conducted by another journal and the authors' response and revisions as well as expedited peer-review in Oncotarget.

\section{REFERENCES}

1. Stewart WF, Ricci JA, Chee E, Morganstein D and Lipton R. Lost productive time and cost due to common pain conditions in the US workforce. JAMA. 2003; 290:24432454.

2. Walker BF. The prevalence of low back pain: a systematic review of the literature from 1966 to 1998. J Spinal Disord. 2000; 13:205-217.

3. Katz JN. Lumbar disc disorders and low-back pain: socioeconomic factors and consequences. J Bone Joint Surg Am. 2006; 88 Suppl 2:21-24.

4. Silva-Correia J, Correia SI, Oliveira JM and Reis RL. Tissue engineering strategies applied in the regeneration of the human intervertebral disk. Biotechnol Adv. 2013; 
31:1514-1531.

5. Di Martino A, Vaccaro AR, Lee JY, Denaro V and Lim MR. Nucleus pulposus replacement: basic science and indications for clinical use. Spine (Phila Pa 1976). 2005; 30:S16-22.

6. Cakir B, Schmidt R, Reichel H and Kafer W. Lumbar disk herniation: what are reliable criterions indicative for surgery? Orthopedics. 2009; 32:589.

7. Ruan D, He Q, Ding Y, Hou L, Li J and Luk KD. Intervertebral disc transplantation in the treatment of degenerative spine disease: a preliminary study. Lancet. 2007; 369:993-999.

8. Paesold G, Nerlich AG and Boos N. Biological treatment strategies for disc degeneration: potentials and shortcomings. Eur Spine J. 2007; 16:447-468.

9. Baer AE, Wang JY, Kraus VB and Setton LA. Collagen gene expression and mechanical properties of intervertebral disc cell-alginate cultures. J Orthop Res. 2001; 19:2-10.

10. Alini M, Li W, Markovic P, Aebi M, Spiro RC and Roughley PJ. The potential and limitations of a cell-seeded collagen/hyaluronan scaffold to engineer an intervertebral disc-like matrix. Spine (Phila Pa 1976). 2003; 28:446-454; discussion 453.

11. Nesti LJ, Li WJ, Shanti RM, Jiang YJ, Jackson W, Freedman BA, Kuklo TR, Giuliani JR and Tuan RS. Intervertebral disc tissue engineering using a novel hyaluronic acid-nanofibrous scaffold (HANFS) amalgam. Tissue Eng Part A. 2008; 14:1527-1537.

12. Yang SH, Wu CC, Shih TT, Sun YH and Lin FH. In vitro study on interaction between human nucleus pulposus cells and mesenchymal stem cells through paracrine stimulation. Spine (Phila Pa 1976). 2008; 33:1951-1957.

13. Takegami K, An HS, Kumano F, Chiba K, Thonar EJ, Singh $\mathrm{K}$ and Masuda K. Osteogenic protein-1 is most effective in stimulating nucleus pulposus and annulus fibrosus cells to repair their matrix after chondroitinase $\mathrm{ABC}$-induced in vitro chemonucleolysis. Spine J. 2005; 5:231-238.

14. Yow SZ, Quek CH, Yim EK, Lim CT and Leong KW. Collagen-based fibrous scaffold for spatial organization of encapsulated and seeded human mesenchymal stem cells. Biomaterials. 2009; 30:1133-1142.

15. Chou AI and Nicoll SB. Characterization of photocrosslinked alginate hydrogels for nucleus pulposus cell encapsulation. J Biomed Mater Res A. 2009; 91:187194.

16. Cloyd JM, Malhotra NR, Weng L, Chen W, Mauck RL and Elliott DM. Material properties in unconfined compression of human nucleus pulposus, injectable hyaluronic acidbased hydrogels and tissue engineering scaffolds. Eur Spine J. 2007; 16:1892-1898.

17. Song JJ and Ott HC. Organ engineering based on decellularized matrix scaffolds. Trends Mol Med. 2011; 17:424-432.

18. Crapo PM, Gilbert TW and Badylak SF. An overview of tissue and whole organ decellularization processes. Biomaterials. 2011; 32:3233-3243.

19. Mercuri JJ, Gill SS and Simionescu DT. Novel tissuederived biomimetic scaffold for regenerating the human nucleus pulposus. J Biomed Mater Res A. 2011; 96:422435.

20. Mercuri JJ, Patnaik S, Dion G, Gill SS, Liao J and Simionescu DT. Regenerative potential of decellularized porcine nucleus pulposus hydrogel scaffolds: stem cell differentiation, matrix remodeling, and biocompatibility studies. Tissue Eng Part A. 2013; 19:952-966.

21. Xu H, Xu B, Yang Q, Li X, Ma X, Xia Q, Zhang Y, Zhang $\mathrm{C}$ and $\mathrm{Wu} \mathrm{Y}$. Comparison of decellularization protocols for preparing a decellularized porcine annulus fibrosus scaffold. PLoS One. 2014; 9:e86723.

22. Chan LK, Leung VY, Tam V, Lu WW, Sze KY and Cheung KM. Decellularized bovine intervertebral disc as a natural scaffold for xenogenic cell studies. Acta Biomater. 2013; 9:5262-5272.

23. Yuan M, Yeung CW, Li YY, Diao H, Cheung KM, Chan D, Cheah K and Chan PB. Effects of nucleus pulposus cell-derived acellular matrix on the differentiation of mesenchymal stem cells. Biomaterials. 2013; 34:39483961.

24. Grauss RW, Hazekamp MG, Oppenhuizen F, van Munsteren CJ, Gittenberger-de Groot AC and DeRuiter MC. Histological evaluation of decellularised porcine aortic valves: matrix changes due to different decellularisation methods. Eur J Cardiothorac Surg. 2005; 27:566-571.

25. Keane TJ, Londono R, Turner NJ and Badylak SF. Consequences of ineffective decellularization of biologic scaffolds on the host response. Biomaterials. 2012; 33:1771-1781.

26. Nord A and Haugejorden O. [Two-year trial of the fluoride-containing varnishes Duraphat and Carex]. Nor Tannlaegeforen Tid. 1991; 101:46-49.

27. Gilbert TW, Sellaro TL and Badylak SF. Decellularization of tissues and organs. Biomaterials. 2006; 27:3675-3683.

28. Tanemura M, Yin D, Chong AS and Galili U. Differential immune responses to alpha-gal epitopes on xenografts and allografts: implications for accommodation in xenotransplantation. J Clin Invest. 2000; 105:301-310.

29. Brown BN, Valentin JE, Stewart-Akers AM, McCabe GP and Badylak SF. Macrophage phenotype and remodeling outcomes in response to biologic scaffolds with and without a cellular component. Biomaterials. 2009; 30:1482-1491.

30. Lee CR, Sakai D, Nakai T, Toyama K, Mochida J, Alini M and Grad S. A phenotypic comparison of intervertebral disc and articular cartilage cells in the rat. Eur Spine J. 2007; 16:2174-2185.

31. Minogue BM, Richardson SM, Zeef LA, Freemont AJ and Hoyland JA. Characterization of the human nucleus pulposus cell phenotype and evaluation of novel marker gene expression to define adult stem cell differentiation. 
Arthritis Rheum. 2010; 62:3695-3705.

32. Yang H, Wu J, Liu J, Ebraheim M, Castillo S, Liu X, Tang $\mathrm{T}$ and Ebraheim NA. Transplanted mesenchymal stem cells with pure fibrinous gelatin-transforming growth factorbetal decrease rabbit intervertebral disc degeneration. Spine J. 2010; 10:802-810.

33. Stoyanov JV, Gantenbein-Ritter B, Bertolo A, Aebli N, Baur M, Alini M and Grad S. Role of hypoxia and growth and differentiation factor-5 on differentiation of human mesenchymal stem cells towards intervertebral nucleus pulposus-like cells. Eur Cell Mater. 2011; 21:533-547.

34. Gantenbein-Ritter B, Benneker LM, Alini M and Grad S. Differential response of human bone marrow stromal cells to either TGF-beta(1) or rhGDF-5. Eur Spine J. 2011; 20:962-971.

35. O'Halloran DM and Pandit AS. Tissue-engineering approach to regenerating the intervertebral disc. Tissue Eng. 2007; 13:1927-1954.

36. Urban JP and Roberts S. Degeneration of the intervertebral disc. Arthritis Res Ther. 2003; 5:120-130.

37. Leung VY, Aladin DM, Lv F, Tam V, Sun Y, Lau RY, Hung SC, Ngan AH, Tang B, Lim CT, Wu EX, Luk KD, Lu WW, Masuda K, Chan D and Cheung KM. Mesenchymal stem cells reduce intervertebral disc fibrosis and facilitate repair. Stem Cells. 2014; 32:2164-2177.

38. Sakai D, Mochida J, Yamamoto Y, Nomura T, Okuma M, Nishimura $K$, Nakai $T$, Ando $K$ and Hotta $T$. Transplantation of mesenchymal stem cells embedded in Atelocollagen gel to the intervertebral disc: a potential therapeutic model for disc degeneration. Biomaterials. 2003; 24:3531-3541.

39. Sakai D, Mochida J, Iwashina T, Hiyama A, Omi H, Imai M, Nakai T, Ando K and Hotta T. Regenerative effects of transplanting mesenchymal stem cells embedded in atelocollagen to the degenerated intervertebral disc. Biomaterials. 2006; 27:335-345.

40. Yoshikawa T, Ueda Y, Miyazaki K, Koizumi M and Takakura Y. Disc regeneration therapy using marrow mesenchymal cell transplantation: a report of two case studies. Spine (Phila Pa 1976). 2010; 35:E475-480.

41. Grunert P, Borde BH, Hudson KD, Macielak MR, Bonassar LJ and Hartl R. Annular repair using high-density collagen gel: a rat-tail in vivo model. Spine (Phila Pa 1976). 2014; 39:198-206.

42. Borde B, Grunert P, Hartl R and Bonassar LJ. Injectable, high-density collagen gels for annulus fibrosus repair: An in vitro rat tail model. J Biomed Mater Res A. 2015; 103:2571-2581.

43. Tim Yoon S, Su Kim K, Li J, Soo Park J, Akamaru T, Elmer WA and Hutton WC. The effect of bone morphogenetic protein-2 on rat intervertebral disc cells in vitro. Spine (Phila Pa 1976). 2003; 28:1773-1780.

44. Uygun BE, Soto-Gutierrez A, Yagi H, Izamis ML, Guzzardi MA, Shulman C, Milwid J, Kobayashi N, Tilles
A, Berthiaume F, Hertl M, Nahmias Y, Yarmush ML and Uygun K. Organ reengineering through development of a transplantable recellularized liver graft using decellularized liver matrix. Nat Med. 2010; 16:814-820.

45. Pei $M$ and He F. Extracellular matrix deposited by synovium-derived stem cells delays replicative senescent chondrocyte dedifferentiation and enhances redifferentiation. J Cell Physiol. 2012; 227:2163-2174.

46. Chen S, Huang Y, Zhou ZJ, Hu ZJ, Wang JY, Xu WB, Fang $\mathrm{XQ}$ and Fan SW. Upregulation of tumor necrosis factor alpha and ADAMTS-5, but not ADAMTS-4, in human intervertebral cartilage endplate with modic changes. Spine (Phila Pa 1976). 2014; 39:E817-825.

47. Chen K, Lin X, Zhang Q, Ni J, Li J, Xiao J, Wang Y, Ye Y, Chen L and Jin K. Decellularized periosteum as a potential biologic scaffold for bone tissue engineering. Acta Biomater. 2015; 19:46-55.

48. Chan BP, Fu SC, Qin L, Rolf C and Chan KM. Pyridinoline in relation to ultimate stress of the patellar tendon during healing: an animal study. J Orthop Res. 1998; 16:597-603.

49. Chen S, Jin G, Huang KM, Ma JJ, Wang Q, Ma Y, Tang XZ, Zhou ZJ, Hu ZJ, Wang JY, Qin A and Fan SW. Lycorine suppresses RANKL-induced osteoclastogenesis in vitro and prevents ovariectomy-induced osteoporosis and titanium particle-induced osteolysis in vivo. Sci Rep. 2015; 5:12853.

50. Shan Z, Li S, Liu J, Mamuti M, Wang C and Zhao F. Correlation between biomechanical properties of the annulus fibrosus and magnetic resonance imaging (MRI) findings. Eur Spine J. 2015; 24:1909-1916.

51. Zhao FD, Pollintine P, Hole BD, Adams MA and Dolan P. Vertebral fractures usually affect the cranial endplate because it is thinner and supported by less-dense trabecular bone. Bone. 2009; 44:372-379.

52. Junhui L, Zhengfeng M, Zhi S, Mamuti M, Lu H, Shunwu F and Fengdong Z. Anchorage of annulus fibrosus within the vertebral endplate with reference to disc herniation. Microsc Res Tech. 2015; 78:754-760.

53. Sobajima S, Kompel JF, Kim JS, Wallach CJ, Robertson DD, Vogt MT, Kang JD and Gilbertson LG. A slowly progressive and reproducible animal model of intervertebral disc degeneration characterized by MRI, X-ray, and histology. Spine (Phila Pa 1976). 2005; 30:15-24.

54. Hu ZJ, Fang XQ, Zhou ZJ, Wang JY, Zhao FD and Fan SW. Effect and possible mechanism of muscle-splitting approach on multifidus muscle injury and atrophy after posterior lumbar spine surgery. J Bone Joint Surg Am. 2013; 95:e192(191-199). 